\title{
Endothelial fibrinolytic reactivity and the risk of deep venous thrombosis after spinal cord injury
}

\author{
L Boudaoud $^{1}$, J Roussi ${ }^{1}$, S Lortat-Jacob ${ }^{1}$, B Bussel ${ }^{1}$, O Dizien ${ }^{1}$ and L Drouet ${ }^{2}$ \\ ${ }^{1}$ Raymond Poincaré Hospital, 104 Boulevard Raymond Poincaré, 92380 Garches and ${ }^{2}$ Lariboisière Hospital, 2 rue \\ Ambroise Paré, Paris, France
}

\begin{abstract}
Deep vein thrombosis (DVT) is a frequent event in patients with spinal cord injury, even with prophylactic anticoagulant therapy. Lower limb paralysis is a known major risk factor for venous thrombosis, supposedly due to the venostasis in relation with total immobility. The main goal of this study was to evaluate the endothelial response to anoxia to determine whether recovery of fibrinolytic potential occurs in patients subjected to forced bedrest because of a spinal cord injury and whether this recovery is related to the incidence and/or evolution of DVT. We evaluated vascular endothelium reactivity in the lower limbs no longer submitted to the hydrostatic pressure of the erected position in 15 patients with paraplegia or tetraplegia and in 10 normal volunteers after venous occlusion produced by the application of $10 \mathrm{~cm} \mathrm{Hg}$ pressure to the lower limb for $15 \mathrm{~min}$ comparatively to the upper limb used as reference. Among the 15 patients, 10 whose spinal cord injury had occurred 1 to 6 months earlier were still receiving prophylactic anticoagulant therapy, whereas the five other patients were not receiving prophylactic anticoagulants because the injury dated back 6 months or more. After venostasis, tissue plasminogen activator (tPA) increased significantly in both patients and controls in the upper limb (tPA levels twofold and threefold respectively in controls and patients) but showed no significant changes in the lower limb; prolonged immobility did not allow recovery in the lower limbs of a level of fibrinolytic responsiveness identical to that in the upper limbs. The plasminogen activator inhibitor $\left(\mathrm{PAI}_{1}\right)$ remained unchanged after anoxia, although wide interindividual variations were seen. Natural coagulation inhibitors and circulating blood stigmates of hypercoagulability were measured. None of the patients had abnormally low levels of coagulation inhibitors (ie, antithrombin III, protein $\mathrm{C}$ and protein $\mathrm{S}$ levels were normal). Seventy-five per cent of patients (prophylactically anticoagulated or not) had very high levels of fibrin degradation products (D. Dimer levels sevenfold to eightfold those of the controls), but all patients had normal levels of thrombinantithrombin complexes and prothrombin fragments $1+2$. The permanence of the thrombotic process characterized by an increase in D. Dimer levels without recovery of fibrinolytic potential suggests a proposal for the patients an indefinite antithrombotic treatment at curative doses.
\end{abstract}

Keywords: venous occlusion; spinal cord injury; thrombosis; fibrinolytic reactivity; D. Dimers

\section{Introduction}

Venous thrombosis is one of the main complications after spinal cord injury, ${ }^{1}$ although its prevalence has varied across studies, in particular as a result of differences in diagnostic methods. Using clinical criteria, the frequent prevalence of deep vein thrombosis (DVT) usually concerning the lower limbs varies from $12 \%$ to $64 \% .^{2-5}$ When labelled fibrinogen, plethysmography or phlebography were used to detect DVT, prevalences of $47 \%$ to $100 \%{ }^{4,6}$ were found in the absence of prophylactic anticoagulant therapy. The incidence of pulmonary embolism ranged from $2 \%$ to $16 \%{ }^{7,8}$ In addition, even when conventional prophy-

Correspondence: L Boudaoud laxis with heparin or an oral vitamin $\mathrm{K}$ antagonist is given, DVT is common in patients with paraplegia or tetraplegia from the acute injury and until the third month after the injury. ${ }^{9-11}$ This frequency of DVT decreases after 6 months, but the DVT is still apparent several months following the injury. ${ }^{11}$

Venous stasis due to muscular hypotonia and prolonged immobility is associated with this high incidence of thrombosis. As endothelial reactivity is modulated by circulating blood flow and hydrostatic pressure, it was interesting to evaluate this reactivity in the lower limbs compared to the upper limbs in these patients whose spinal cord injury had occurred 1 to 6 months earlier and to compare to controls. 
It has been demonstrated that in normal individuals, fibrinolytic activity is lower in the lower limbs than in the upper limbs, ${ }^{12,13}$ and that this difference was due to physiological adaptation to the venous hydrostatic pressure to which the lower limbs are subjected during standing and to the effects of exhaustion. In a previous study, ${ }^{14}$ we showed that in normal subjects, global endothelial reactivity elicited by venostasis was greater in the upper limbs than in the lower limbs, among which the left leg seemed to be the less reactive, probably linked to anatomical conditions inducing an additional stasis condition and explaining why DVT is more common in the lower limbs, especially the left leg. Fibrinolytic reactivity of the veins of upper limbs tested after venous occlusion had been applied to the upper arm was found to be decreased in paraplegic individuals during the first few days after injury ${ }^{15}$ and correlated with the increased risk of venous thrombosis. Recovery of normal fibrinolytic activity of the lower limbs during prolonged forced bedrest has been observed $^{13}$ in eleven patients 12 to 33 days after the injury, fracture of one femoral shaft or pelvis without spinal cord injury, but there has not been a study of paraplegic or tetraplegic patients with at least 1 month following the onset of the paralysis. The aim of our work, performed in 15 patients with paraplegia or tetraplegia, was:

- to evaluate if the vascular endothelium in the lower limbs recovers function similar to that of the upper limbs after a long period of forced bedrest (1 to 6 months) by determination of plasmatic levels of the von Willebrand factor (vWF), tissue plasminogen activator (tPA), tissue plasminogen activator inhibitor $\left(\mathrm{PAI}_{1}\right)$ both before and after an experimental venostasis;

- to measure markers for coagulation activation and thrombolysis and to test their diagnostic value;

- to determine the activity of physiological coagulation inhibitors in patients with spinal cord injury.

\section{Patients and methods}

Subjects

Fifteen male spinal cord injury patients aged 18 to 60 years agreed to participate in the study. Ten patients whose spinal cord injuries had occurred 1 to 6 months earlier were receiving prophylactic anticoagulant therapy (nine were receiving low molecular weight heparin and one an oral vitamin $\mathrm{K}$ antagonist); the other five patients whose injuries dated back 6 months or more were not receiving prophylactic anticoagulants. All participants gave their written informed consent before they were entered into the trial.

Ten healthy male volunteers aged 28 to 55 years participated in the study. None had received anticoagulant, antiinflammatory, antibiotic, phlebotonic, or vasculoptropic medications within 30 days before the study.
The study was conducted in accordance with the terms of the Declaration of Helsinki (revised in Hong Kong in 1989) and with the French law $\mathrm{n}^{\circ} 88.1138$ and its application decree dated 27 Sept. '90 on the protection of persons who participate in biomedical research.

\section{Sampling protocol}

To verify the stability and reproducibility of study parameters, the test was done twice at a 1 week interval (D0 and D7) in the healthy volunteers and at a 2 month interval (D0-D60) in the spinal cord injury patients.

Blood was always sampled at $10 \mathrm{~h} 00 \mathrm{am}$ to minimize any circadian variations. Before sampling, each subject ate a carbohydrate-based breakfast and rested for at least $30 \mathrm{~min}$. Samples were collected from an antecubital vein in the upper limb and from a vein of the leg or from the dorsum of the foot.

A variant of the venous occlusion test described by Robertson $^{12}$ was used. In the controls, a blood pressure cuff inflated at $100 \mathrm{mmHg}$ was used to produce venous occlusion in the right upper and lower limbs for $15 \mathrm{~min}$. The left upper limb served as the control. Samples were drawn sequentially from the left upper limb prior to venous occlusion and from the right upper limb then the right lower limb after $20 \mathrm{~min}$ rest and after $15 \mathrm{~min}$ venous occlusion. In the patients, the same procedure was used in the upper limbs, the lower limbs (right or left) which were studied were free of venous thrombosis as judged by clinical criteria and doppler ultrasonography or phlebography.

\section{Test and methods}

Venous blood samples were obtained using a Vacutainer (Becton Dickinson). Samples for blood cell and platelet counts were collected in tubes containing EDTA $(0.072 \mathrm{~mL}, 0.17 \mathrm{M})$. Diatubes containing 9 volumes of blood for 1 volume of anticoagulant (citrate $0.11 \mathrm{M}$, theophylline $15 \mathrm{nM}$, adenosine $37 \mathrm{nM}$, and dipyramidole $0.198 \mathrm{nM}$, Diagnostica Stago) were used for assays of hemostasis parameters. Serum proteins were determined on blood collected in gel (inert gel). Platelet-free plasma was obtained by centrifuging blood samples at $4000 \mathrm{~g}$ for $20 \mathrm{~min}$ within $1 \mathrm{~h}$ of sampling. Plasma samples were separated in $300 \mu \mathrm{l}$ aliquots and stored at $-80^{\circ} \mathrm{C}$. The following parameters were measured:

- blood cell and platelet counts (on an automated counter, Argos ABX, Roche);

- fibrinogen (Fg) (von Clauss method), protein S (PS), antithrombin III (ATIII) and protein C (PC) using chronometric or chromogenic method with adapted reagents and on an STA automate from Diagnostica Stago;

- tissue plasminogen activator (tPA), plasminogen activator inhibitor $1\left(\mathrm{PAI}_{1}\right)$, von Willebrand factor 
(vWF), and fibrin degradation products (D. Dimers) using an immunoenzymometric assay (ELISA) and Asserachrom specific reagents (Diagnostica Stago);

- prothrombin fragments $1+2\left(\mathrm{~F}_{1+2}\right)$, thrombinantithrombin complex (TAT) using an immunoenzymometric assay (ELISA) and Enzygnost specific reagents (Behring);

- serum proteins on a nephelometer (Behring) with Behring adaped reagents.

Statistical analysis was achieved using the Statgraphics system (Uniware) with Student's $t$ test.

\section{Results}

Reproducibility

All the parameter values (tPA, $\mathrm{PAI}_{1}, \mathrm{vWF}, \mathrm{F}_{1+2}$, TAT, D. Dimers, hematocrit, and serum protein) measured in the upper and lower limbs before and after venous occlusion showed no significant difference between D0 and D7 in the controls. Values on D0 and D7 were, therefore, considered equivalent.

Similarly, in the spinal cord injury group, there were no significant differences between D0 and D60, and the two sets of values were, therefore, considered equivalent (Table 1).

Table 1 Parameter values in the upper and lower limbs before and after venous occlusion in the controls (C) and in spinal cord injury patients $(\mathrm{P})$

\begin{tabular}{lcccc}
\hline & & $\begin{array}{c}\text { Before VO } \\
\text { UL }\end{array}$ & $\begin{array}{c}\text { After } \\
\text { UL }\end{array}$ & LO \\
\hline $\mathrm{Ht}$ & $\mathrm{C}$ & $42.6 \pm 0.4$ & $48.3 \pm 0.4^{* * *}$ & $45.8 \pm 0.7^{*}$ \\
$\%$ & $\mathrm{P}$ & $36.8 \pm 1$ & $41.2 \pm 1.3^{* * *}$ & $37.9 \pm 1.1$ \\
$\mathrm{Prot}$ & $\mathrm{C}$ & $66.7 \pm 3.1$ & $80.6 \pm 2.5^{* * *}$ & $73.7 \pm 2.9$ \\
$\mathrm{~g} / \mathrm{l}$ & $\mathrm{P}$ & $66.9 \pm 1.4$ & $77.7 \pm 2.5^{* * *}$ & $69.3 \pm 1.6$ \\
$\mathrm{Fg}$ & $\mathrm{C}$ & $2.1 \pm 0.1$ & $2.3 \pm 0.1$ & $2.2 \pm 0.1$ \\
$\mathrm{~g} / \mathrm{l}$ & $\mathrm{P}$ & $3.9 \pm 0.2$ & $4.8 \pm 0.3$ & $4.2 \pm 0.3$ \\
$\mathrm{tPA}$ & $\mathrm{C}$ & $6.4 \pm 1.1$ & $12.7 \pm 1.3^{* * *}$ & $9.4 \pm 2.5$ \\
$\mathrm{ng} / \mathrm{ml}$ & $\mathrm{P}$ & $8 \pm 2.7$ & $27.3 \pm 7.5^{* *}$ & $13.8 \pm 4.3$ \\
$\mathrm{PAI}$ & $\mathrm{C}$ & $20.4 \pm 6.4$ & $18.8 \pm 6.2$ & $27.2 \pm 8.4$ \\
$\mathrm{ng} / \mathrm{ml}$ & $\mathrm{P}$ & $7.8 \pm 2.4$ & $9.2 \pm 2.2$ & $6.2 \pm 2.4$ \\
$\mathrm{vWF}$ & $\mathrm{C}$ & $103.5 \pm 5.8$ & $128 \pm 7.7$ & $112.8 \pm 8.4$ \\
$\%$ & $\mathrm{P}$ & $109 \pm 8.3$ & $123 \pm 7.2$ & $118.5 \pm 9.4$ \\
$\mathrm{~F}$ & $\mathrm{C}$ & $0.96 \pm 0.06$ & $0.99 \pm 0.06$ & $1.5 \pm 0.4$ \\
$\mathrm{ng} / \mathrm{ml}$ & $\mathrm{P}$ & $0.7 \pm 0.1$ & $0.9 \pm 0.3$ & $1.1 \pm 0.3$ \\
$\mathrm{TAT}$ & $\mathrm{C}$ & $4.3 \pm 1.3$ & $5.2 \pm 1.7$ & $4.5 \pm 1.5$ \\
$\mathrm{ng} / \mathrm{ml}$ & $\mathrm{P}$ & $4.1 \pm 0.9$ & $7.6 \pm 2.5$ & $3.7 \pm 0.5$ \\
$\mathrm{D} . \mathrm{Di}$ & $\mathrm{C}$ & $88.6 \pm 12.2$ & $147.4 \pm 26.6$ & $112.9 \pm 17.2$ \\
$\mathrm{ng} / \mathrm{ml}$ & $\mathrm{P}$ & $884.5 \pm 183$ & $1013.5 \pm 192$ & $830 \pm 164$ \\
\hline
\end{tabular}

Mean \pm standard error; UL: upper limb; LL: lower limb; VO: venous occlusion; C: Normal controls; P: spinal cord injury patients; $\mathrm{Ht}$ : hematocrit; Prot: serum proteins; Fg: fibrinogen; tPA: tissue plasminogen activator; $\mathrm{PAI}_{1}$ : plasminogen activator inhibitor 1; vWF: von Willebrand factor; $\mathrm{F}_{1+2}$ : prothrombin fragments $1+2$; TAT: thrombinantithrombin complex; D. Dimers: fibrin degradation products. $* P<0.05 ; * * P<0.01 ; * * * P<0.001$
Efficacy of venous occlusion (Figure 1)

The efficacy of venous occlusion was evaluated on the basis of parameters reflecting hemoconcentration, ie, hematocrit, serum proteins and Fg levels. Hematocrit, serum proteins and $\mathrm{Fg}$ levels increased in the upper limb in both groups, by $13 \pm 1 \%, 20 \pm 3 \%$ and $10 \pm 4 \%$, respectively in the controls and by $12 \pm 3 \%$, $16 \pm 3 \%$ and $23 \pm 7 \%$, respectively in the patients. The increase in hematocrit and serum proteins were statistically significant but not the Fg level increase. No significant increase in these parameters was seen in the lower limb: hematocrit, serum proteins and fibrinogen rose by $7.5 \pm 1.5 \%, 10 \pm 4 \%$ and $5 \pm 4 \%$, respectively in the controls and by $3 \pm 3 \%, 4 \pm 2 \%$ and $8 \pm 7 \%$, respectively in the patients.

Effect of venous occlusion on endothelial cells release Marked rises of tPA levels were seen in the upper limb after stasis, twofold in controls $(6.4 \pm 1.1$ to $12.7 \pm$ $1.3 \mathrm{ng} / \mathrm{ml})$, threefold in patients $(8 \pm 2.7$ to $27 \pm 7.5 \mathrm{ng} /$ $\mathrm{ml}$ ), whereas lower limb values did not increase either in the controls or in the patients. No tendency towards an increase in releasable tPA was observed as a function of time. Post-stasis levels of tPA at the upper limb were higher in the patients than in the controls. The opposite post-stasis levels of tPA in the lower limb were no different from pre-stasis either in the patients or in the controls (Figure 2). Stasis had no effect on $\mathrm{PAI}_{1}$ levels. tPA and $\mathrm{PAI}_{1}$ were similar in patients with and without DVT, both before and after stasis (Figure 2).

Trend towards increases in von Willebrand factor levels were seen: in the controls, values rose by $24 \pm 6 \%$ and $8 \pm 7 \%$ in the upper limb and lower limb respectively, versus $12 \pm 6 \%$ and $8 \pm 7 \%$ in the upper limb and lower limb in the spinal cord injury patients; however, the differences failed to reach statistical significance as a result of wide inter-individual variations.

\section{Markers for activation of coagulation}

Levels of TAT and $\mathrm{F}_{1+2}$ were normal in the two groups and were not modified by venous occlusion. In contrast, D. Dimers levels were eight times higher in the patient's group than in the controls. All individual D. Dimers values in resting patients were higher than values in the controls. Levels were very high, and there was considerable inter-individual variation. No time effect was found for D. Dimers (Figure 3). In both the patients and the controls, there was a trend towards higher post-stasis values in the upper limbs as compared to the lower limbs, but the difference was not statistically significant.

\section{Correlation between DVT occurrence and D. Dimer levels}

Among the 15 spinal cord injury patients, three were diagnosed with DVT by Doppler ultrasonography $(n=1)$ or phlebography $(n=2)$. All of the patients with DVT had a very high level of $\mathrm{D}$. Dimers and none of the 

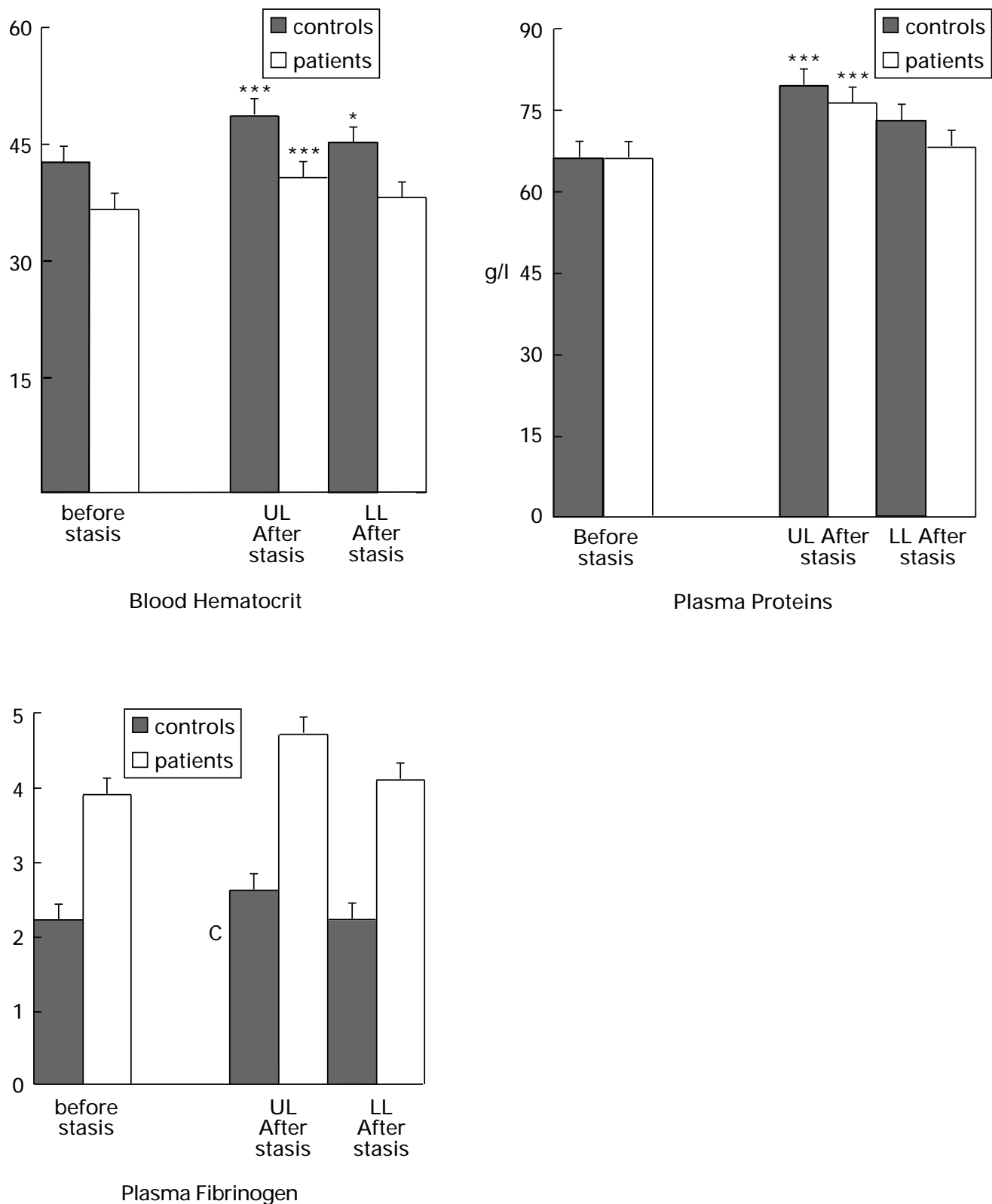

Figure 1 Effects of venous occlusion on parameters reflecting hemoconcentration; Hematocrit; Plasma Proteins; Plasma Fibrinogen; UL: upper limb; LL: lower limb; $* P<0.05$; $* * P<0.01 ; * * * P<0.001$

patients with D. Dimers lower than $500 \mathrm{ng} / \mathrm{ml}$ had DVT. Six patients with D. Dimers higher than $700 \mathrm{ng} / \mathrm{ml}$ and five patients more than $1000 \mathrm{ng} / \mathrm{ml}$ had no diagnosticable DVT (Figure 4).

No difference was observed in D. Dimer levels between the 10 patients prophylactically treated by low molecular weight heparin and the five patients who were not on prophylactic therapy.

Inflammation-related proteins and coagulation inhibitors The levels of haptoglobin, orosomucoid, and fibrinogen in the spinal cord injury patients were increased as compared with normal subjects. Comparison of the inflammation-related proteins was based on the time that had elapsed since the accident, that is the period of bedrest from 1 to 6 months. No significant time effect was found except for a slight tendency toward a decrease with time in these factors (Figure 5).

Activities of physiological coagulation inhibitors (AT III, PC, and PS) were normal in the spinal cord injury group (Table 2). One patient received an oral vitamin $\mathrm{K}$ antagonist and his PC and PS were proportionally decreased to other vitamin $\mathrm{K}$ dependant proteins. 


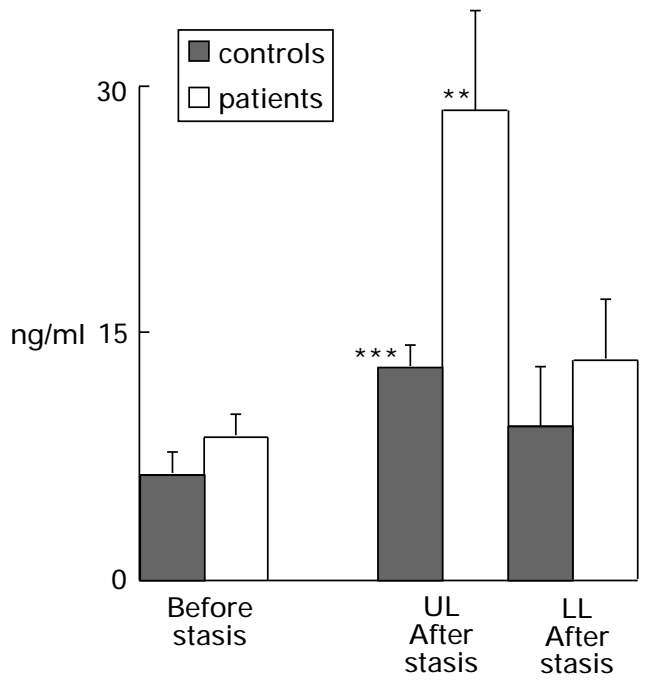

a: tPA

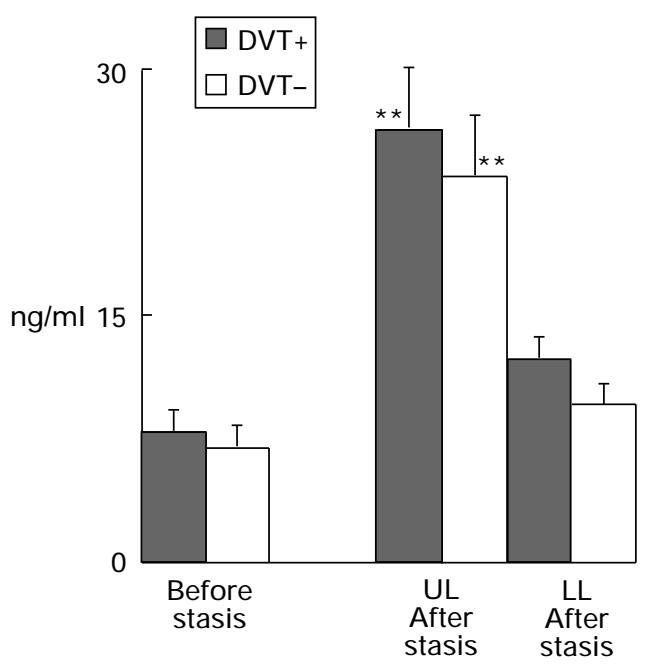

b: tPA

Figure 2 Effects of venous occlusion on released tPA by endothelial cells. (a) comparison between controls and patients; (b) comparison between patients with or without documented DVT; UL: upper limb; LL: lower limb; $* P<0.05$; **P<0.01; $* * * P<0.001$

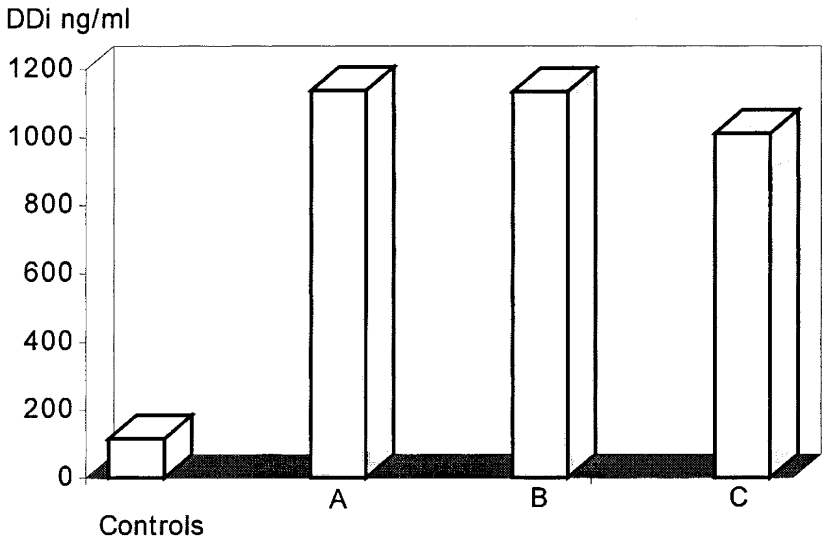

Figure 3 Time effect and D. Dimer levels. (a) patients studied in early time ( 0 to 1 month). (b) middle time (1 to 6 months). (c) late time ( $>6$ months)

\section{Discussion}

The aim of our work was to study the haemostatic function of patients with spinal cord injury and particularly the fibrinolytic pathway, the endothelial reactivity and the activation of coagulation. A method widely used to investigate fibrinolysis is the venous occlusion test, ${ }^{12}$ which allows evaluation of endothelial reactivity in a segment of a limb subjected to venous occlusion. ${ }^{14,16,17}$ Using this test, decreased fibrinolytic capacity measured in the upper limb has been found in $35 \%$ of patients with recurrent thromboembolism. ${ }^{18}$ The venous occlusion test is typically applied to the upper limbs but in this case we have adapted the test to

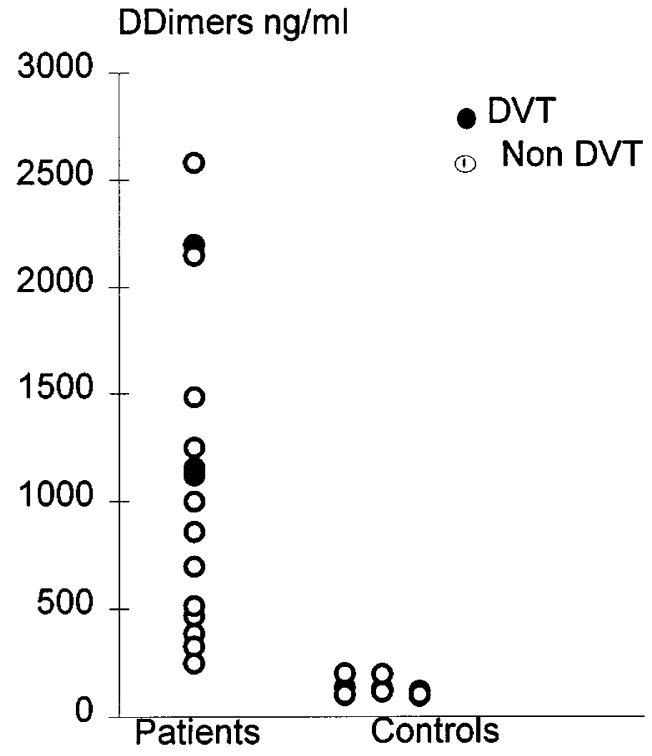

Figure 4 Individual values of D. Dimers in Controls and Patients with no $(\bigcirc)$ or documented $(\bullet)$ DVT

study the reactivity of the veins of the lower limbs. Thus, venous occlusion using similar pressures produces different effects in the upper and in lower limbs, characterized by a release of lower amounts of endothelial factors (such as tPA) in the lower limbs than in the upper limbs in healthy volunteers ${ }^{14}$ as well as in spinal cord injury patients contrary to Keber's study which shows a recovery of fibrinolytic activity in spinal cord injury patients. ${ }^{13}$ In normal subjects, it has been hypothesized that the differences observed 
Table 2 Inflammation-related proteins and coagulation inhibitors

\begin{tabular}{lcccccc}
\hline & $\begin{array}{c}\text { Hapto } \\
g / l\end{array}$ & $\begin{array}{c}\text { Oroso } \\
g / l\end{array}$ & $\begin{array}{c}F g \\
g / l\end{array}$ & $\begin{array}{c}A T I I I \\
\%\end{array}$ & $\begin{array}{c}P C \\
\%\end{array}$ & $\begin{array}{c}P S \\
\%\end{array}$ \\
\hline Normal & & & & & & \\
Controls & $1.8 \pm 0.4$ & $0.83 \pm 1.1$ & $2.2 \pm 0.1$ & $107.5 \pm 9$ & $111 \pm 7.8$ & $105 \pm 10$ \\
Patients & $3 \pm 0.5$ & $1.3 \pm 0.1$ & $3.9 \pm 0.2$ & $117 \pm 2.8$ & $125 \pm 7.8$ & $99.7 \pm 6$ \\
\hline
\end{tabular}

Mean value \pm SE; UL: upper limb; LL: lower limb; Hapto: haptoglobin; Fg: fibrinogen; Oroso: orosomucoid; ATIII: antithrombin; PC: protein C; PS: protein $S$

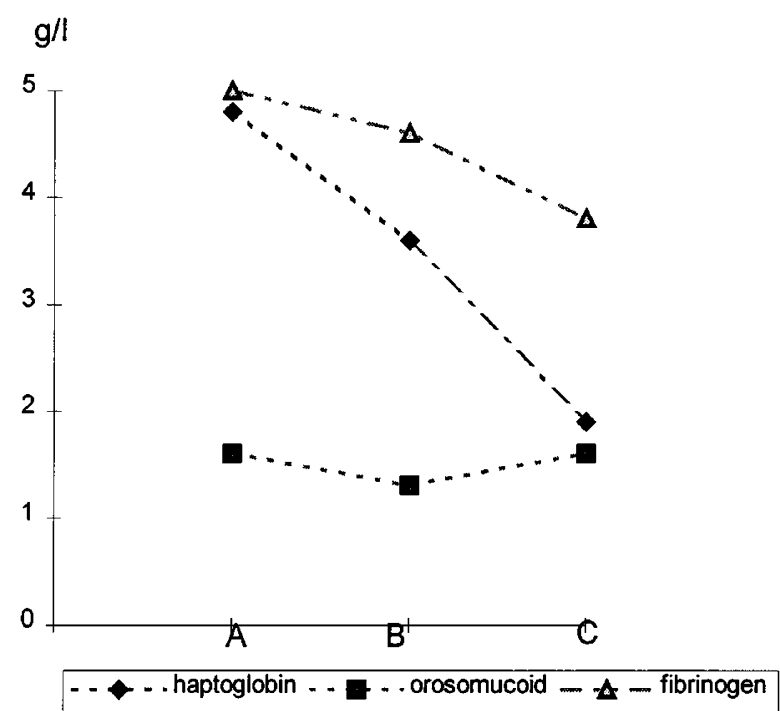

Figure 5 Time effect and Inflammation-related proteins. (a) patients studied in early time (0 to 1 months). (b) middle time (1 to 6 months). (c) late time ( $>6$ months)

between the upper and lower limbs could be acquired and ascribable to exhaustion of lower limb endothelial cells due to the hydrostatic pressure produced by the upright position, which is considerably higher in the lower limbs than in the upper limbs. The fact that paraplegic patients who have forced bedrest react as in the normal controls does not go against this hypothesis.

Another hypothesis to explain the lack of response to venous stasis in the lower limb could be the effectiveness of venous stasis (indicated by the slightest increase in the hemoconcentration parameters) due to the fact the larger muscular masses in the lower limbs cannot be compressed as efficiently as in the upper limb. The fact that paraplegic individuals whose muscle loss is significant react as do normal controls does not go against this hypothesis. The lack of induced hemoconcentration led us to assume that permeability of the capillary endothelium in the lower limb is lower than the capillary endothelium in the upper limb. Again, there was no discrepancy between the normal controls and the patients. Moreover, in a preliminary study, the $\mathrm{PO}_{2}$ was measured before and after the venous stasis found in the normal controls. This study showed that in spite of the lower hemoconcentration, the same level of desaturation, and therefore the same level of venous stasis was found in the lower limb.

Results of thrombotic marker assays in the patients showed that D. Dimer levels ${ }^{19}$ were increased sevenfold as compared with the controls, whereas the levels of TAT and $\mathrm{F}_{1+2}$ were normal in controls and in patients. Elevation of D. Dimer levels denotes activation of fibrin formation inside and even outside the vascular system and therefore indicates that clinical or subclinical thrombosis has occurred despite the administration of prophylactic anticoagulant therapy. Is the increase of D. Dimer levels without an increase of TAT and $F_{1+2}$ levels linked to the effect of therapy which even if it is not completely efficient does not slow down D. Dimer levels increase, while TAT andF $F_{1+2}$ are normal? High D. Dimer levels can also be due to extravascular thrombin formation in areas of ischemia related to pressure sores, which are common in spinal cord injury patients and favoured by the period of bedrest and oedema of lower limbs and sometimes heterotopic ossification. ${ }^{20}$

Different sets of factors associated with an increased risk of DVT are present in spinal cord injury patients. Venous stasis due to forced bedrest and absence of muscle contractions, inflammation, frequently present in spinal cord injury patients, are known to promote venous thrombosis. ${ }^{21,22}$ The absence of recovery of fibrinolytic potential results in loss of protection against thrombosis. This combination of risk factors explains why prophylactic anticoagulant therapy fails to provide complete protection from DVT in patients with spinal cord injury. In our study, three patients developed DVT despite having anticoagulant treatment. Most of the patients without DVT had very high D. Dimer levels and no difference between D. Dimer levels was observed. These data suggest that conventional therapy with low molecular weight heparin was inadequate. Some studies ${ }^{23}$ found that individuallytailored doses of heparin (APTT $\times 1.5$ times control) were more effective in preventing DVT in paraplegic patients than were fixed-doses of 5000 UI given subcutaneously twice daily; DVT was detected respectively in $7 \%$ versus $31 \%$ of patients. 


\section{Conclusion}

This study demonstrates that prolonged bedrest was not associated with the recovery of fibrinolytic activity since no tendency was found towards an increase in the rate of releasable tPA in patients who have been bedridden for 6 months as compared to those patients whose accident occurred later (1 month ago). This fact implies that anticoagulant therapy has to be more powerful. As D. Dimer levels are significantly increased in thromboembolisms but also in other conditions such as pressure sores which are also frequent in paraplegic patients, a study comparing coagulation markers, in particular D. Dimer levels and doppler ultrasonography findings in a large group of paraplegic patients would provide additional information on the sensitivity of D. Dimer levels for the diagnosis of DVT.

\section{Acknowledgements}

This research was supported by 'The Foundation Garches'.

\section{References}

1 Chiles BW, Cooper PR. Acute spinal injury. New Engl J of Med 1996; 334: $514-520$.

2 Walsh JJ, Tribe C. Phlebo-thrombosis and pulmonary embolism in paraplegia. Paraplegia 1965; 3: $209-213$.

3 Silver JR, Mouton A. Prophylactic anticoagulant therapy against pulmonary embolism in acute paraplegia. $\mathrm{Br}$ Med J 1970; 2: $338-340$.

4 Myllynen $\mathrm{P}$ et al. Deep venous thrombosis and pulmonary embolism in patients with acute spinal cord injury: comparison with non paralysed patients immobilized due to spinal fractures. J Trauma 1985; 25: $541-543$.

5 Weingarden SI. Deep venous thrombosis in spinal cord injury: overview of the problem. Chest 1992; 102: 636S-639S (suppl).

6 Brach BB et al. Venous thrombosis in acute spinal cord paralysis. J Trauma 1977; 17: 289-292.
7 Perkash A, Prakash V, Perkash I. Experience with the management of thromboembolism in patients with spinal cord injury. Part I: Incidence, diagnosis and role of some risk factors. Paraplegia 1979; 16: 322 - 331 .

8 Waring WP, Karunas RS. Acute spinal cord injuries and the incidence of clinically occurring thromboembolic disease. Paraplegia 1991; 29: 8-16.

9 Yelnik A et al. Accidents thrombo-emboliques chez les paraplégiques: intérêt et limites du traitement anticoagulant préventif. Actualités en rééducation fonctionnelle et réadaptation 1986; $11^{\mathrm{e}}$ série Masson (ed.) 1986; pp. 75-82 Paris.

10 Hachnen HJ. Anticoagulant therapy in patients with spinal cord injury. Paraplegia 1974; 12: $176-187$.

11 Green D et al. Prevention of thromboembolism in spinal cord injury: role of low molecular weight heparin. Arch Phys Med Rehabil 1994; 75: 290-292.

12 Robertson BR, Pandolfi M, Nilsson IM. 'Fibrinolytic capacity' in healthy volunteers as estimated from effect of venous occlusion of arms. Act Chir Scand 1972; 138: 429-436.

13 Keber D. The increase of leg fibrinolytic potential after reduction of hydrostatic stimulus. Tromb Haemostas 1983; 50: 731 - 734 .

14 Baillart et al. Differences between upper and lower limbs in venous endothelial reactivity in humans. Phlebology 1994; suppl. 1: $10-14$.

15 Petaja J, Myllinen P, Rokkanen P, Nokelainen M. Fibrinolysis and spinal injury. Relationship to post-traumatic deep vein thrombosis. Acta Chir Scand 1989; 155: 241 - 246.

16 Wiman B et al. The role of fibrinolytic system in deep vein thrombosis. J Lab Clin Med 1985; 105: 267.

17 Jennings I, Luddington RJ, Harper PL. Changes in endothelial related coagulation proteins in response to venous occlusion. Thromb Haemost 1991; 65: 374.

18 Juhan-Vague I et al. Deficient tPA release and elevated PA Inhibitor levels in patients with spontaneous or recurrent deep venous thrombosis. Thromb Haemost 1987; 57: 67-72.

19 Bounameaux H, De Moerloose P, Perrier A, Reber A. Plasma measurement of D-Dimer as diagnostic aid in suspected venous thromboembolism: an overview. Thromb Haemost 1994; 71: 1 -6.

20 Perkash A et al. Persistent hypercoagulation associated with heteroptopic ossification in patients with spinal cord injury long after injury has occurred. Paraplegia 1993; 31: 653-659.

21 Nicolaides AN, Kakkar VV, Fields ES, Fish P. Venous stasis and deep vein thrombosis. Br. J. Surg 1972; 59: 713-716.

22 Yelnik A et al. Systematic lower limb phlebography in acute spinal cord injury in 147 patients. Paraplegia 1991; 29: $253-260$.

23 Green D et al. Fixed-vs-adjusted-dose heparin in the prophylaxis of thromboembolism in spinal cord injury. JAMA 1988; 260: $1255-1258$. 